\title{
Hoffmann-Ocon, Andreas
}

\section{"Keine passiven Redekränzchen". Soziale und organisatorische Experimente in der politischen Kultur der Lehrerpersonen(fort)bildung in Zürich um 1918}

De Vincenti, Andrea [Hrsg.]; Grube, Norbert [Hrsg.]; Hoffmann-Ocon, Andreas [Hrsg.]: 1918 in Bildung und Erziehung. Traditionen, Transitionen, Visionen. Bad Heilbrunn : Verlag Julius Klinkhardt 2020, S. 109-134. - (Historische Bildungsforschung)

Quellenangabe/ Reference:

Hoffmann-Ocon, Andreas: "Keine passiven Redekränzchen". Soziale und organisatorische Experimente in der politischen Kultur der Lehrerpersonen(fort)bildung in Zürich um 1918 - In: De Vincenti, Andrea [Hrsg.]; Grube, Norbert [Hrsg.]; Hoffmann-Ocon, Andreas [Hrsg.]: 1918 in Bildung und Erziehung. Traditionen, Transitionen, Visionen. Bad Heilbrunn : Verlag Julius Klinkhardt 2020, S. 109-134 - URN: urn:nbn:de:0111-pedocs-204669 - DOI: 10.25656/01:20466

https://nbn-resolving.org/urn:nbn:de:0111-pedocs-204669 https://doi.org/10.25656/01:20466

in Kooperation mit / in cooperation with:

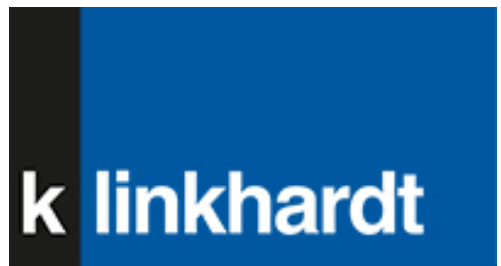

http://www.klinkhardt.de

\section{Nutzungsbedingungen}

Dieses Dokument steht unter folgender Creative Commons-Lizenz: http://creativecommons.org/licenses/by-nc-sa/4.0/deed.de - Sie dürfen das Werk bzw. den Inhalt unter folgenden Bedingungen vervielfältigen, verbreiten und öffentlich zugänglich machen sowie Abwandlungen und Bearbeitungen des Werkes bzw. Inhaltes anfertigen: Sie müssen den Namen des Autors/Rechteinhabers in der von ihm festgelegten Weise nennen. Dieses Werk bzw. der Inhalt darf nicht für kommerzielle Zwecke verwendet werden. Die neu entstandenen Werke bzw. Inhalte dürfen nur unter Verwendung von Lizenzbedingungen weitergegeben werden, die mit denen dieses Lizenzvertrages identisch oder vergleichbar sind.

Mit der Verwendung dieses Dokuments erkennen Sie die Nutzungsbedingungen an.

\section{Terms of use}

This document is published under following Creative Commons-License: http://creativecommons.org/licenses/by-nc-sa/4.0/deed.en - You may copy, distribute and transmit, adapt or exhibit the work in the public and alter, transform or change this work as long as you attribute the work in the manner specified by the author or licensor. You are not allowed to make commercial use of the work. If you alter, transform, or change this work in any way, you may distribute the resulting work only under this or a comparable license.

By using this particular document, you accept the above-stated conditions of use.

\section{Kontakt / Contact:}

peDOcs

DIPF | Leibniz-Institut für Bildungsforschung und Bildungsinformation

Informationszentrum (IZ) Bildung

E-Mail: pedocs@dipf.de

Internet: www.pedocs.de

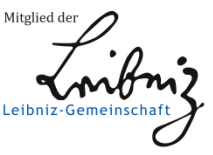




\section{Historische Bildungsforschung}

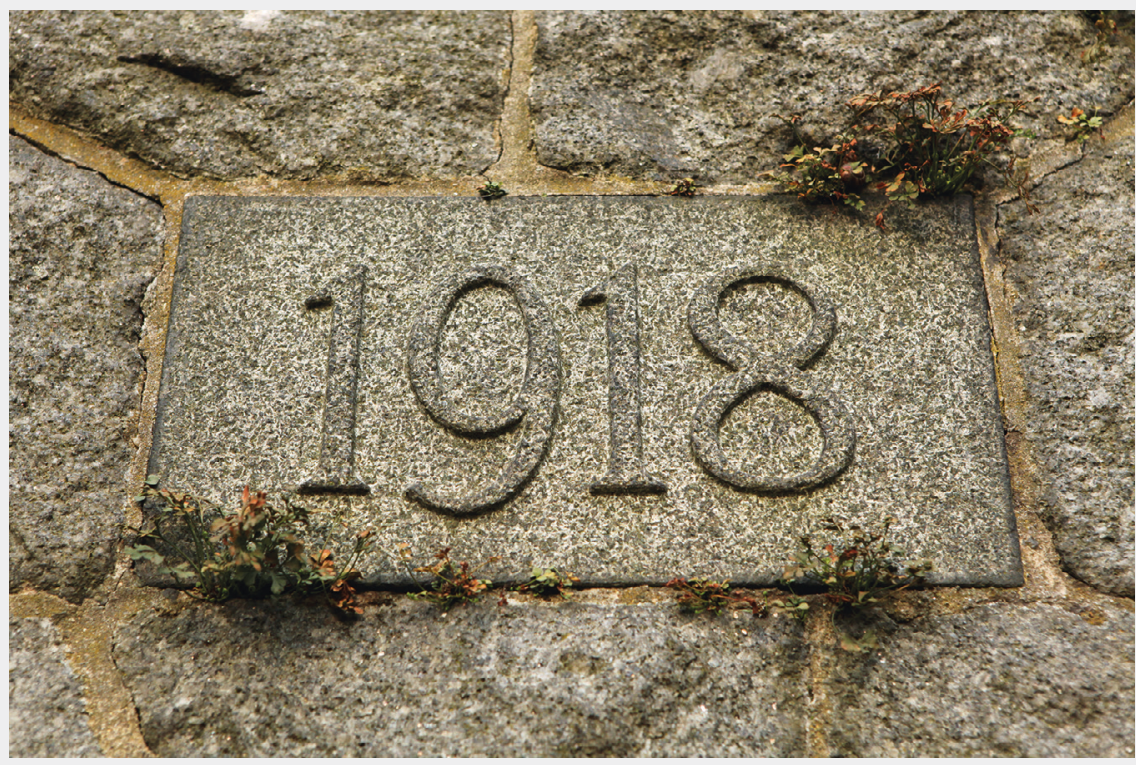

\section{Andrea De Vincenti Norbert Grube}

Andreas Hoffmann-Ocon (Hrsg.)

1918 in Bildung und Erziehung

Traditionen, Transitionen, Visionen 
De Vincenti / Grube / Hoffmann-Ocon 1918 in Bildung und Erziehung 


\section{Historische Bildungsforschung}

Tagungsbände der Sektion

Historische Bildungsforschung

herausgegeben vom Vorstand der

Sektion Historische Bildungsforschung

der Deutschen Gesellschaft für Erziehungswissenschaft (DGfE) 


\section{Andrea De Vincenti \\ Norbert Grube Andreas Hoffmann-Ocon (Hrsg.)}

\section{8 in Bildung und Erziehung}

Traditionen, Transitionen, Visionen 
Gefördert mit Mitteln der Pädagogischen Hochschule Zürich und der Sektion Historische Bildungsforschung der DGfE.

Dieser Titel wurde in das Programm des Verlages mittels eines Peer-Review-Verfahrens aufgenommen. Für weitere Informationen siehe www.klinkhardt.de.

Bibliografische Information der Deutschen Nationalbibliothek Die Deutsche Nationalbibliothek verzeichnet diese Publikation in der Deutschen Nationalbibliografie; detaillierte bibliografische Daten sind im Internet abrufbar über http://dnb.d-nb.de.

2020.i. (c) by Julius Klinkhardt.

Foto Umschlagseite 1: (c) by Vladimir Wrangel / Adobe Stock.

Druck und Bindung: AZ Druck und Datentechnik, Kempten.

Printed in Germany 2020.

Gedruckt auf chlorfrei gebleichtem alterungsbeständigem Papier.

Die Publikation (mit Ausnabme aller Fotos, Grafiken und Abbildungen) ist veröffent-

licht unter der Creative Commons-Lizenz: CC BY-NC-SA 4.0 International https://creativecommons.org/licenses/by-nc-sa/4.0/

ISBN 978-3-7815-5827-4 Digital

doi.org/10.35468/5827

ISBN 978-3-7815-2395-1 Print 


\section{Inhaltsverzeichnis}

Andrea De Vincenti, Norbert Grube und Andreas Hoffmann-Ocon

Die Chiffre 1918. Aushandlungen und Konflikte um Konzeptionen,

Visionen und Transitionen in Bildung und Erziehung

\section{Jugend - Ambivalenzen zwischen Aufbegehren, Bewegung und Tradiertem}

Jennifer Burri und Adrian Juen

Schule zwischen Harmonie und Aufstand - SchülerInnenmitbestimmung an den Zürcher LehrerInnenseminaren um 1918

Andrea De Vincenti

Patriotische Männlichkeiten? Körper- und Charaktererziehung im Seminar-Turnverein Küsnacht 1900-1925

Elija Horn

Jugendbewegung und „Indien“: Zur Herausbildung eines

jugendkulturellen Topos' um 1918 87

Schule und Pädagogik - Infragestellung, Re-Assortierung und Weitergabe von Wissen

Andreas Hoffmann-Ocon

„Keine passiven Redekränzchen“ - Soziale und organisatorische

Experimente in der politischen Kultur der Lehrerpersonen(fort)bildung in Zürich um 1918

\section{Tomáš Kasper}

Die wissenschaftliche Neuorientierung der tschechischen Pädagogik im Windschatten ihres amerikanischen Vorbildes Eine Fallstudie zur Zwischenkriegszeit 
Viktoria Luise Gräbe

Schulbücher für den evangelischen Religionsunterricht und die

Lebenskunde in der Weimarer Republik -

Zwischen Kontinuität und Aufbruch

Sozialdemokratie, Sozialismus und Pädagogik -

Verflechtungen in Wien, Zürich und der Deutschschweiz

Norbert Grube

Lehrer, Politiker, „Trämlergeneral“, Schriftsteller: Der Zürcher

Sozialdemkorat Alfred Traber als Beispiel für dynamisierte

Pädagogisierungsschübe um 1918

\section{Lucien Criblez}

Bildungs- und Erziehungsaspirationen der politischen Linken in der

Schweiz in den 1910er- und 1920er-Jahren

Wilfried Göttlicher

Otto Glöckels Schulreform, das Rote Wien und die deutsche

Reformpädagogik. Zur Einordnung der Glöckelschen

Schulreform, 1919-1934

Ulrich Herrmann

Transitionen und Visionen für das Bildungs- und Schulwesen im

Bundesland Wien am Beginn der ersten österreichischen Republik

Autorinnen und Autoren 


\section{Andreas Hoffmann-Ocon}

\section{„Keine passiven Redekränzchen“" Soziale und organisatorische Experimente in der politischen Kultur der Lehrpersonen(fort)bildung in Zürich um 1918*}

Dass die Zeit nach dem Ersten Weltkrieg bis in die Mitte der 1920er-Jahre als eine eigene Epoche verstanden werden kann, wird immer wieder betont. ${ }^{1}$ In der Schweiz dominierten neben Themen zur Armut, zur Spanischen Grippe und ihren sozialen Auswirkungen die politischen Folgen des Landesstreiks die öffentliche Debatte. ${ }^{2}$ Soziale Spannungen waren Impulsgeber unterschiedlicher Deutungen und prägten Abspaltungen und Lagerbildung innerhalb der sozialistischen Bewegung. In Zürich dienten Streiks als Bezugspunkte verschiedener politischer Parteien, selbst Bankangestellte ließen sich im Herbst 1918 zu Arbeitsniederlegungen mobilisieren. ${ }^{3}$ Inwiefern diese sozialen Umbruchsituationen als kennzeichnend für den Zeitabschnitt des Kriegsendes gedeutet werden können, bleibt fraglich. So ordnet etwa Jakob Tanner die historische Beschreibung der Landesstreikphase im Sinne eines nationalen Traumas als Stilisierung ein und versteht die konstruierten Kontinuitätslinien, etwa die Zusammenschaltung vom Ersten und Zweiten Weltkrieg, als Teil der Herstellung eines kulturellen Gedächtnisses in der Schweiz. ${ }^{4}$

In der Geschichtsschreibung wird der Erste Weltkrieg oftmals als Katalysator gedeutet, der die Öffnung von Regierungssystemen, Organisationen und Gremien beschleunigen konnte, so dass neue Unsicherheiten und neue Möglich-

\footnotetext{
* Dieser Beitrag entstand im Rahmen des SNF-Projekts 166008 ,Wissenschaft - Erziehung und Alltag. Orte und Praktiken der Zürcher Primarlehrer/innenbildung in der ersten Hälfte des 20. Jahrhunderts".

1 Vgl. Schönpflug 2017, S. 307.

2 Vgl. Buomberger 2014; zum Streit über die Verelendung der Massen als ein Auslöser des Landesstreiks vgl. Tribelhorn 2018.

3 Vgl. Zala 2014, S. 498.

4 Vgl. Tanner 2014, S. 9 f.
} 
keiten nach 1918 den (bildungs-) politischen Handlungshorizont strukturierten. ${ }^{5}$ Die Betonung von Öffnung und Offenheit problematisiert eine Sichtweise, ,die die Jahre zwischen 1914 und 1945 zu einem ununterbrochenen und zusammenhängenden Konfliktgeschehen erklärt“" und einen ,direkte[n] Weg vom Ersten zum Zweiten Weltkrieg" nahelegt. ${ }^{6}$ Der Beitrag soll an diesen Befunden von Unsicherheiten und Möglichkeiten anknüpfen und am Beispiel der bisher wenig erforschten Zürcher Schulkapitel nach der Stabilität und Fragilität von Formen und Kulturen der Lehrpersonen(fort)bildung um 1918 fragen. Das von Lehrpersonen weitgehend selbstorganisierte Gremium „Schulkapitel“ wird mit seinen widersprüchlichen Verschiebungen von nebeneinander gestellten Wissenselementen aus Pädagogik, Didaktik, Politik und empirisch angereicherten Wissenschaften als ein eigensinniges, streckenweise assoziativ funktionierendes „Erkenntnismodell“ betrachtet. $^{7}$ Die politische Kultur der Lehrpersonen soll in einer moderat ausgeprägten praxeologischen Perspektivierung ermittelt werden. So geht es darum, Handlungsroutinen und Brüche von Handlungsroutinen zu beschreiben, Regelwissen zu ermitteln, damalige Kontingenzspielräume zu erfassen ${ }^{8}$ und sich dafür zu interessieren,

wie sich soziale Ordnungen [...] durch je situativ spezifische Ausformungen sozialer Praxis konstituieren und ebenso dafür, wie diese verworfen werden, sich neu formieren $[\ldots]$ und sich wiederum fortschreiben, stabilisieren oder verändern. ${ }^{9}$

Rückgekoppelt zum Kulturbegriff bedeutet dies in dem hier präsentierten $\mathrm{Zu}$ sammenhang, dass Kultur nicht als abgeschlossenes Ganzes begriffen wird, sondern als „Ensemble von Praktiken, Orientierungen und Ordnungen sozialen Verhaltes“, welches „Einstellungen und habitualisierte Verhaltensweisen mit normativen Charakter" umfassen kann. ${ }^{10}$

Aus Sicht einflussreicher Zeitgenossen in der Lehrpersonenbildung hatten sich 1918 die Sektionen der Zürcher Schulkapitel überlebt: „Die Sektionen sollen aufgehoben werden" ${ }^{11}$ lautete eine gereizt vorgetragene Forderung. Zu klären ist, ob solche Quellenhinweise entscheidende Reorganisations- oder gar Demokratisierungsschübe in der Lehrpersonenbildung markieren. Ein Teil der Mitglieder nahm die Schulkapitel als zu behördlich und hierarchisch organisiert war und forderte eine flexiblere Aufnahme von „Protestthemen“ zu um-

\footnotetext{
Vgl. Tanner 2015, S. 159; Müller/Tooze 2015.

6 Janz 2013, S. 334f.

7 Vgl. Hoffmann-Ocon 2016a, S. 252.

8 Vgl. Graf 2008, S. 119.

9 Freist in: Haasis/Rieske 2015b, S. 207.

${ }^{10}$ Jaeggi 2014, S. 77.

${ }^{11}$ Forschungsbibliothek Pestalozzianum (FBP), Prot. Schulkapitel Hinwil, 11.05.1918, S. 351.
} 
fassenden Bildungs- und Sozialreformen. ${ }^{12}$ Der in diesem Beitrag eingeschlagene Weg setzt bei Debattenrekonstruktionen, Vorstellungen und Erwartungen sowie Selbst- und Situationswahrnehmungen der mehrheitlich, jedoch nicht ausschließlich männlichen Schulkapitelmitglieder an. Zu der quellenorientierten Annäherung gehört ebenfalls die kontextsensible Erschließung der Akteurskonstellationen in ihren bildungspolitischen Praxiszusammenhängen, in ihren zeitgenössischen Handlungsrahmen und Erwartungshorizonten. Die Erschließung von Debattenpraktiken in Gremien soll auch für Spuren in den Quellen offen sein, die auf ein „Getriebensein“ hinweisen, das Menschen in Gruppen unter sich erzeugen und welches - wie es Christian Meier nennt - die Verfeinerung des historischen Bildes durch eine ,prozessuale Betrachtungsweise" anmahnt. ${ }^{13}$

Die angestrebten und zur Diskussion gestellten interpretativen Vorschläge nehmen sowohl „Vorgeschichten“ der vermeintlichen Reorganisationsschübe, zum Beispiel durch besonders exponierte Vorträge, als auch die damals bereits reflektierten Mobilisierungsbeschränkungen auf, wie etwa Abwesenheit an Versammlungen aufgrund von Grippeangst, die in der Schweiz durch schlechte medizinische Versorgung zu teilweise hilflosen Reaktionen geführt hatte. ${ }^{14}$ Dass zentrale Lehrpersonen - etwa der 1918 neben seinem Lehrberuf auch als Mitarbeiter und späterer Sekretär bei der Jugendfürsorge-Stiftung Pro Juventute tätige und zunächst sozialistisch gesonnene Emil Jucker - ihren Ausgangspunkt im charismatischen bildungspolitischen Handeln suchten, ${ }^{15}$ sollte im Hinblick auf die Programmatik der Schulkapitel, deren Protagonisten sowohl Partizipations- als auch Pädagogisierungsabsichten verfolgten, als Spannungsfeld befragt werden. $\mathrm{Ob}$ die sich verschiebenden alltäglichen Praktiken in den Schulkapiteln als durch den langen Schatten des Ersten Weltkriegs imprägniert, durch einen zukunftsoptimistischen Aufbruch oder gar durch beide sich vermischende Orientierungen zu verstehen sind, soll analysiert werden und ein Feld für Anschlussfragen eröffnen. Die Hauptquellen bilden Protokolle einzelner Schulkapitel und Schulsynodalversammlungen, die regelmäßig zu den mehrmals im Jahr stattfindenden Treffen verfasst wurden und in der Forschungsbibliothek Pestalozzianum (FBP) und dem Staatsarchiv Zürich verwahrt werden. Sie beinhalten unter anderem bildungs- und standespolitische Berichte, Referate zu pädagogischen, didaktischen, gesellschaftlichen und kulturellen Fragen sowie Notizen zum Debattenverlauf. Weiterhin wurden Ver-

\footnotetext{
12 Luhmann 1996, S. 184.

13 Meier 2014, S. 25.

14 Vgl. Kreis 2014, S. 228.

15 Vgl. Desiderato/Lengwiler/Rothenbühler 2008, S. 14.
} 


\section{2 | Andreas Hoffmann-Ocon}

sammlungsprotokolle der Sozialdemokratischen Lehrervereinigung Zürichs aus dem Schweizerischen Sozialarchiv (SOZARCH) in Zürich herangezogen.

\section{Schulkapitel - Institutionen und Praxen}

Die institutionalisierten Schulkapitel im Kanton Zürich ermöglichten es den Lehrpersonen, sich in einem Gruppenzusammenhang von regionalen Konferenzen fortzubilden. Die gegen Mitte des 19. Jahrhunderts gegründeten Einrichtungen sollten jedoch nicht nur der Fortbildung, sondern auch der mehr bildungspolitischen Vorbereitung von Synodalgeschäften dienen. Die Schulsynode wiederum galt als ständische Organisation der Zürcher (Volksschul-) Lehrerschaft mit ausgedehnten Rechten gegenüber der schulpolitischen Verwaltung. ${ }^{16}$ Der korporative Charakter der Schulkapitel zeigte sich formal vor allem darin, dass eine geheime Wahl des Vorstandes und eine Ernennung der dieser Institution zugestandenen Mitgliedern der Bezirksschulpflegen (kommunale Schulaufsichtsbehörden) und Abgeordneten für die Prosynode stattfand. Vier ordentliche Versammlungen sollten jährlich gehalten werden; der Präsident konnte außerordentliche Zusammenkünfte einberufen. ${ }^{17}$ Einige Schulkapitel, insbesondere in den Städten Zürich und Winterthur, differenzierten sich noch in weitere Abteilungen, Kreise und Teilsektionen aus, so dass es, je nach Periode, zwischen 10 und 12 große Kapitelkonferenzen (Affoltern, Andelfingen, Bülach, Dielsdorf, Hinwil, Horgen, Pfäffikon, Uster, Winterthur, Zürich) und 10 weitere Teilkapitelkonferenzen gab.

Aus einer praxeologischen Perspektive ist erwähnenswert, dass die jeweiligen Schulkapiteltreffen an einem Samstagvormittag oftmals in wechselnden Gastwirtschaften stattfanden, wenn diese nicht, wie im Bezirk Horgen und Affoltern, auch in Schulhäusern durchgeführt wurden. Wird der durch die Teilnehmenden bildungspolitisch und pädagogisch angeeignete Raum, der außerhalb des eigentlichen Volksschulsystems lag, als ein spezielles Medium betrachtet, ${ }^{18}$ stellt sich die Frage, ob und wie die Tonalität der Debatten, Gespräche sowie die Artikulationsweisen von diesem Setting beeinflusst waren. ${ }^{19}$ Wird überdies die Debatte im Wirtshaus als Praxis und Performanz der Lehrpersonenfortbildung weitergedacht, inkludiert dies eine spezifische Wissensproduktion. Der Schauplatz des Wirtshauses als tradierte Drehscheibe für neue politische Ideen und Obrigkeitskritik zwang den Schulkapitelmitgliedern ein ande-

\footnotetext{
${ }^{16}$ Vgl. Hoffmann-Ocon 2016a, S. 252.

${ }^{17}$ Vgl. Kreis 1933, S. 438; Hoffmann-Ocon 2018, S. 55.

${ }^{18}$ Vgl. Kraus 2005, S. 7.

${ }^{19}$ Vgl. Mersch 2015, S. 11; zum Wirtshaus als Ort des Austausches, der Selbstdarstellung und -positionierung: vgl. den Beitrag von Andrea De Vincenti in diesem Band.
} 
res Sehen auf. ${ }^{20}$ Der stets wechselnde Raum und Versammlungssaal im Wirtshaus stellte eine Art Bühne dar, auf der an die vorangegangenen Geschehnisse erinnert wurde, aber allein schon durch wechselnde Betrachterpositionen auch Irritation oder Unruhe gestiftet werden konnte. Nicht in dem Sinne, dass die aufgesuchten Räume und Versammlungssäle in ihrer Materialität allein konstitutiv für die jeweiligen Debatten gewesen wären, jedoch als „Übergangszonen zwischen innen und außen", ${ }^{21}$ dürften sie einen diffusen Einfluss auf den Gesprächsverlauf gehabt haben.

So waren in der Institution der Schulkapitel Praktiken eingelagert, die auf komplexen Kooperationsbeziehungen fußten: In den Versammlungen konkurrenzierten sich Gruppen mit verschiedenen bildungspolitischen Positionen, teilten sich aber in ständig neu ausgehandelten Arrangements den Einflussbereich der Schulkapitel zu zukünftigen Weichenstellungen im Bereich der Volksschulbildung. Der Schulkapitelalltag konnte streckenweise dem „Typ“ einer sozialen Bewegung entsprechen. Der Ansatzpunkt vieler Debatten zielte nicht nur auf die Veränderung des (Lehrer- oder Schüler-)Individuums, sondern auch auf die Veränderung der Gesellschaft und Sozialstruktur. ${ }^{22}$

\section{Indikatoren einer Krise der Schulkapitel?}

Welche Indikatoren deuteten eine (Legitimations-)Krise der Schulkapitel resp. der Konstitution der Schulkapitel an? Aus Sicht von „Bewahrern“23 der Schulkapitel in ihrer bisherigen Form verbarg sich eine tiefere Gefährdung in den zerbröckelten Gewissheiten, in welchen Formen zukünftig Lehrpersonen ausgebildet werden sollten. Die Frage nach einer Erneuerung der Schulkapitel während und nach dem Ersten Weltkrieg ging im Kanton Zürich mit der Frage nach Erneuerung der Lehrpersonenbildung einher, die ihren Ausdruck in verschiedenen Orten für angehende Primarlehrpersonen fand - eine Situation, die für die meisten an Lehrpersonenbildung Beteiligten aus ganz unterschiedlichen Gründen als unbefriedigend eingeschätzt wurde. ${ }^{24}$ Neben unterschiedlich religiös-konfessionellen, staatlichen und privaten sowie speziell an Frauen gerichteten Seminarorten, gab es seit 1907 die Möglichkeit, nachmaturitär in Form eines einjährigen Kurses an der Universität ein Lehrdiplom zu erlangen. Welche Bedeutung und Stellung die Schulkapitel und die ihr nachgelagerte Schulsynode für die organisierte Lehrerinnen- und Lehrerschaft haben könnten, was

\footnotetext{
${ }^{20}$ Graber 2017, S. 191.

${ }^{21}$ Binotto 2013, S. 163.

${ }^{22}$ Raschke 1985, S. 107.

${ }^{23}$ Tyack/Cuban 1995, S. 13.

${ }^{24}$ Hoffmann-Ocon 2018, S. 55.
} 


\section{4 | Andreas Hoffmann-Ocon}

die Einflussnahme auf die Schulgesetzgebung und die Lehrerbildungsreform anging, waren offene Fragen in einer bildungspolitischen Selbstverständigungsdebatte unter den Lehrpersonen.

\subsection{Konkurrierende Orte bildungspolitischer und pädagogischer Debatten}

Kritisch wurde während und nach Ende des Ersten Weltkriegs die fehlende Kompetenzaufteilung zwischen den „Konferenzen“ im Sinne von Teil- und Unterkapiteln, Schulkapiteln sowie Schulsynode betrachtet. Alle diese Gremien beanspruchten für sich, der geeignete Ort für die Diskussion und Abklärung von Erziehungsfragen und ,für die rechtzeitige Berücksichtigung geistiger Strömungen in ihrer Bedeutung für die öffentliche Erziehung zu sein“. ${ }^{25}$ An der Schwelle zum 20. Jahrhundert gewannen neben Schulkapiteln und Schulsynode andere Foren der bildungspolitischen und pädagogischen Debatte an Bedeutung. Insbesondere Lehrerverbände und Fachvereine, die sich ohne formale Aufsicht durch den Erziehungsrat beweglicher und dynamischer Bildungsfragen annehmen konnten, übernahmen aus funktionaler Sicht ebenfalls Aufgaben, die zuvor in den Schulkapiteln und der Schulsynode behandelt wurden. ${ }^{26}$ Die Zeit vor und nach dem Ersten Weltkrieg brachte eine Vielfalt an Lehrerverbänden hervor, die jeweils ihre Differenz zu den anderen zelebrierten. So schloss sich etwa der eigenständige Schweizerische SeminarlehrerVerein (SSLV) 1915 dem Verein Schweizerischer Gymnasiallehrer (VSG) an, auch um Lohnforderungen der Lehrerschaft in den Krisenjahren und in Zeiten des Kaufkraft-Verlustes besser vertreten zu können. ${ }^{27}$ Wohl durch ein umfassendes Verständnis des Sozialen, Politischen und Pädagogischen mitbedingt, artikulierten Lehrpersonen ihre bildungspolitischen Vorstellungen nicht nur in den Schulkapiteln, sondern auch in anderen Bezügen, etwa in der 1906 gegründeten sozialdemokratischen Lehrervereinigung Zürichs. Der vom Zürcher Sekundarlehrer Jakob Wegmann initiierte Verein diskutierte bildungspolitische Themen, oftmals im Zusammenhang mit der sozialen Frage, und organisierte Bildungsanlässe sowie auch die gemeinsame Lektüre wissenschaftlicher und sozialkritischer Literatur. ${ }^{28}$ Eher abweichend von den Schulkapitelversammlungen befassten sich die Mitglieder der sozialdemokratischen Lehrervereinigung aber auch mit Fragen zur Stellung der Frau in der Gesellschaft, was si-

\footnotetext{
${ }^{25}$ Gassmann 1934, S. 37.

${ }^{26}$ Vgl. Gassmann 1933, S. 620; Keller 1934, S. 29.

27 Vgl. Metz 2017, S. 16f.

${ }^{28}$ Vgl. Protokollbuch, Soz.-dem. LV, SOZARCH, Ar 201.252.1, [07.11.] 1906, S. 5.
} 
cherlich mit der hohen Anzahl von Lehrerinnen an diesem Debattenort zu tun hatte. ${ }^{29}$

Den Protokollen der sozialdemokratischen Lehrervereinigung zufolge wurden bereits 1916 in den Versammlungen Themen sowohl der Schulsynode als vor allem auch der Schulkapitel besprochen und vorberaten. Aber die Ausweitung der politischen Mitwirkung wurde nicht nur durch Vorberatungen lanciert, sondern auch durch den Versuch, systematisch Schulkapitelvorstände aus den Reihen der sozialdemokratischen Lehrervereinigung zu gewinnen. ${ }^{30}$ Das Protokoll der im September 1918 erfolgten „Vorbesprechung der Wahlen in Schulkapitel und Bezirksschulpflege" gibt Aufschluss darüber, dass im Kapitel II von Zürich ,für die Chargen Vice-Präsident und Aktuar [...] die Genossen Leemann und Dr. Oprecht [...] genannt werden, für das Kapitel III werden genannt Manz und Egg, Ersteren nur für Präsident ev. Vice-Präsident ${ }^{\text {“ }}{ }^{31}$ Hans Oprecht etwa schloss 1914 seine Ausbildung zum Primarlehrer im Seminar Küsnacht $a b$, studierte neben seiner Lehrertätigkeit in der Stadt Zürich und Niederglatt noch Psychologie an der Universität Zürich mit dem Abschluss eines Doktorats und wurde in den 1920er-Jahren Nationalrat für die Sozialdemokratische Partei der Schweiz (SPS), in den 1930er-Jahren unter anderem Präsident der SPS und Vorstand der Sozialistischen Internationale. Aus heutiger Sicht zählt er zu einer der führenden Persönlichkeiten der Arbeiterbewegung in der Zwischenkriegszeit, die den Verband der Gemeinde- und Staatsarbeiter (später: VPOD) als Bildungsorganisation mitausbaute. ${ }^{32}$ So konnte die bildungspolitische und pädagogische Interessenbearbeitung an verschiedenen Orten stattfinden, die je mit eigener Autonomie und Handlungsrationalitäten ausgestattet, aber durch „,vagabundierende“ Akteure lose miteinander gekoppelt waren. Das kommunikative Wechselverhältnis zwischen sozialdemokratischem Lehrerverein und Schulkapiteln führte durch einzelne Akteure, die mindestens an beiden Orten wirkten, zu Verflechtungen und bildungspolitisch horizontalen Handlungslogiken. Folgt man der Lesart von Emil Gassmann zu Beginn der 1930er-Jahre, dann standen die Schulkapitel direkt nach dem Ersten Weltkrieg für einen Teil der Lehrpersonen zur Disposition, so dass ihre „Beseitigung“ oder „Ersetzung“" gefordert wurde. ${ }^{33}$

\footnotetext{
${ }^{29}$ Vgl. Protokollbuch, Soz.-dem. LV, SOZARCH, Ar 201.252.1, 10.12.1906, S. 19-22.

${ }^{30}$ Vgl. Protokollbuch, Soz.-dem. LV, SOZARCH, Ar 201.252.1, 06.10.1916, S. 223.

${ }^{31}$ Protokollbuch, Soz.-dem. LV, SOZARCH, Ar 201.252.1, 02.09.1918, S. 268.

${ }^{32}$ Vgl. Bürgi 2010.

33 Vgl. Gassmann 1934, S. 37.
} 


\section{2 „Grippefurcht“" - Schulkapitel als gefährdete und riskante „Gesamtkörper"?}

Neben einer institutionellen Krise, die sich in konkurrierenden Orten der bildungspolitischen Vertretung von Lehrpersonen manifestieren konnte, hinterließ insbesondere das Jahr 1918 mit seiner Grippewelle auch Spuren in den Schulkapiteln. Sie machte den Körper von Lehrpersonen zum „gefährdeten Körper", der, in Zeiten einer Pandemie, dem sozialen Arrangement von Schulklassen ,ausgesetzt“, zum „riskanten Körper“ werden konnte, welcher einmal infiziert, wiederum für andere Zusammenkünfte eine Gefahr bedeutete. ${ }^{34}$ Die Wucht der Spanischen Grippe, für Schweizer Behörden zunächst schwer erkennbar, beeinträchtige das öffentliche Leben stark, so dass Schulen geschlossen oder der Schulanfang verschoben wurde. ${ }^{35}$

Im Bezirk Horgen tagte im Sekundarschulhaus Richterswil die Versammlung der Schulkapitelteilnehmer Ende November 1918 ,im Zeichen der Grippe und der Grippefurcht, denn nur eine kleine Zahl von Kollegen hat sich dazu eingefunden“. ${ }^{36}$ Dass die „Grippefurcht“ nicht unbegründet war, betonten Einträge aus dem Nekrologe-Buch des Schulkapitels. So wurde an der Kapitelversammlung im Herbst 1919 in Horgen durch ein Mitglied dem 26-jährig an den Folgen der Spanischen Grippe verstorbenen Sekundarlehrer Karl Sutter gedacht. Das Basisnarrativ in dieser Rede war Rettung und Erlösung eines jungen vielversprechenden Pädagogen:

Gewissenhaft bereitete er sich auf die Schulstunden vor und erlabte sich dabei in der Musik, indem er seiner Violine lauten Jubel entlockte. [...] Da riss das Schicksal mit rauher [!] Hand den schönen Traum entzwei. Schon im Sommer 1918 erkrankte er [...] an der Grippe. [...]. Am Tage vor dem Kapitel liess er seinen Kollegen melden, er könne nicht mitkommen, er habe stark Fieber. Heimreisen konnte er nicht mehr; seine Mutter musste ihn in Oberrieden pflegen. Die Krisis einer Lungenentzündung, die sich zur Grippe gesellt hatte(,) überwand er glücklich \& schien auf dem Weg zur Genesung zu sein. An seinem Geburtstage schmückten Schülerinnen in froher Hoffnung sein Krankenzimmer mit Blumen. Da starb er plötzlich; geronnenes Blut war in sein Herz gekommen und hatte die schwache Lebenskraft gebrochen. - Auf dem Kirchhof der Realp liegt er begraben, allen Enttäuschungen \& Bitterkeiten des Lebens für immer enthoben. ${ }^{37}$

\footnotetext{
${ }^{34}$ Vgl. Schroer 2005, S. 39f.

${ }^{35}$ Vgl. Senn 2018, S. 41. Welche Handlungsspielräume nichtmilitärische, kantonale, kommunale, kirchliche und gemeinnützige Akteure durch die Zuspitzung der sozialen Lage vorgefunden hatten resp. wahrnahmen, bleibt nach Einschätzung von Christian Sonderegger und Andreas Tscherrig ein Forschungsdesiderat (2016, S. 282).

${ }^{36}$ Schulkapitel Horgen, 30.11.1918, FBP, ZH HC III, 2, 2 a.

${ }^{37}$ Schulkapitel Horgen Nekrologe, Karl Sutter (1893-1919), FBP, ZH HC III 2, 1 b.
} 
Karl Sutter überlebte nicht, aber als zentrale Figur des Nekrologs wurde der als gewissenhaft beschriebene Pädagoge zum Geretteten und auch Erlösten. Aus bereits einer ausweglos erscheinenden Situation wird er (durch die Pflege seiner Mutter) gerettet, um in dramatischer Verdichtung sich für das Fehlen in der Schulkapitelversammlung entschuldigend doch noch kurz danach zu sterben. Mit der Umschreibung: ,allen Enttäuschungen \& Bitterkeiten des Lebens für immer enthoben“" wurde die unerträgliche Gegenwart durch einen Erlösungsgedanken mit einer besseren, nun transzendenten Zukunft verbunden. ${ }^{38}$ Sutter gehörte zu dem Teil der Bevölkerung, der überraschenderweise am stärksten von der Spanischen Grippe betroffen war: Männer im zweiten und dritten Lebensjahrzehnt, Personen, die eigentlich zum körperlich widerstandsfähigsten Teil der Bevölkerung gehören. ${ }^{39}$ Wird der Körper als eine ,unhintergehbare Größe für die Erklärung des Sozialen“ angeführt, ${ }^{40}$ und angenommen, dass die Organisation des Schulfeldes auf die Sichtbarkeit individueller Körper angewiesen war und ist, befanden sich die Schulkapitel 1918/19 als „Gesamtkörper“, der auf die „politisch geformten Körper“ setzte, ${ }^{41}$ in einem problematischen Zustand.

Der Rettungs- und Erlösungsgedanke spielte auch außerhalb von Nekrologen in der Schweiz gegen Ende des Ersten Weltkriegs in Zeiten sozialer und kultureller Umbruchsituationen eine große Rolle: Eine Quelle für den damals häufig genutzten Topos „Rettung“ bildete die Schrift Die neue Schweiz vom religiös-sozialistisch inspirierten katholischen Theologen Leo Ragaz, in der recht unspezifisch die Jugend aufgefordert wurde, ,alte Geistesbequemlichkeiten“ abzulegen und intellektuelle sowie moralische Eigenständigkeit gegenüber als nichtschweizerisch wahrgenommene geistige Strömungen (wieder) zu erlangen. ${ }^{42} \mathrm{Ob}$ und wie religiös-sozialistische Wissenspräsentationen sich mit Debatten in den Schulkapiteln verquickten, wird zu einem späteren Zeitpunkt aufgegriffen.

\section{Gremienpraxis zwischen Institutionen-Vertrauen und Strukturkritik}

Trotz der soeben skizzierten Krisenzeit waren für die Mitglieder der Schulkapitel die über mehrere Jahrzehnte wenig angetasteten Strukturen des Gremiums für Fortbildung und bildungspolitische Orientierung zugleich gegeben und gemacht. Gegeben, da die Angemessenheit bestimmter Gremienpraktiken kaum

\footnotetext{
${ }^{38}$ Vgl. Lehmann/Thüring 2015, S. 14.

${ }^{39}$ Vgl. Sonderegger/Tscherrig 2016, S. 266; Senn 2018, S. 40.

${ }^{40}$ Schroer 2005, S. 8.

${ }^{41}$ Nigro 2015, S. 35.

${ }^{42}$ Vgl. Ragaz 1918, S. 36f.
} 
zur Diskussion stand. Vorträge, vorbereitet von eingeladenen Gästen oder Schulkapitelmitglieder, zentrierten sich - in heutiger Terminologie - um schulund berufsfeldnahe Themen, wie etwa Ueber jugendliches Verbrechertum, Die Schule im Kampf gegen Tuberkulose oder Unsere Demokratie. Als gemacht und damit als herstellbar wurden die Strukturen betrachtet, wenn auch der durch gemeinsame Interpretation gestiftete Zusammenhang, die gemeinsame Ausübung von Praktiken und die geteilte Deutung der Praktiken nicht mehr richtig funktionierten, ${ }^{43}$ gestört wurden oder die Handlungsroutinen an die Kommunikationsoberfläche gerieten. Schon im Frühjahr 1913 schien sich jedoch eine Krise der Kapitel anzudeuten: „In gewohnter Art wurde auch dieses Jahr in den verschiedenen Sectionen gearbeitet", berichtete Sekundarlehrer Bühler aus Wetzikon und bestätigte die Handlungsroutine im Schulkapitel, führte jedoch weiter aus,

dem Absenzenverzeichnis ist zu entnehmen, dass die Reihen der Wissensdurstigen sich beständig lichteten. Dieser Grund [...] hatte hauptsächlich zur Folge, dass der Beschluss gefasst wurde, für das laufende Jahr die Sektionsversammlungen aufzuheben und an deren Stelle eine vierte Kapitelsversammlung abzuhalten. ${ }^{44}$

In dem Protokoll wird das bereits in der Einleitung erwähnte Schulkapitelmitglied Emil Jucker genannt, der in der Versammlung ausführt, dass ihm die jetzige Organisation nicht gefalle und er sich von einer Neueinteilung, vielleicht einer solchen nach Interessengruppen, mehr verspreche. Die Schulkapitel scheinen in dieser Zeit für Jucker eine bedeutsame Bühne dargestellt zu haben, sein Wirken schien in dem Schulkapitel zentral als Katalysator für verschiedene Themen gewesen zu sein. Mit seinen Beiträgen, die sich sowohl auf Organisations- und Strukturfragen als auch auf gesellschaftliche Strömungen wie etwa Anarchismus oder Fürsorge-Bewegung beziehen konnten, eröffnete er in dem Schulkapitel Hinwil verschiedene Denkwege. ${ }^{45}$ Die Debatten um die äußere Ordnung bedingten für die Schulkapitelmitglieder eine Herausbildung von Wissensfeldern. Die neuen Erkenntnisse, die sich womöglich für ein bildungspolitisches Kraftfeld auch als ein Zuwachs an Bedeutsamkeit und Macht für die Lehrpersonen herausstellen konnten, durften etwas kosten:

Einen schönen Zug des Strebens nach stetiger Fortbildung bezeugten unsere Kapitularen, indem sie sich bereit erklärten, aus ihrer eigenen Tasche dazu beizutragen,

\footnotetext{
${ }^{43}$ Vgl. Jaeggi 2014, S. 106.

${ }^{44}$ Schulkapitel Hinwil, 24.05.1913, FBP, ZH HC III 4, 5, S. 267.

${ }^{45}$ Vgl. Hoffmann-Ocon 2016a, S. 262.
} 
um hin und wieder einen tüchtigen, auswärtigen Referenten in unsern Versammlungen anhören zu können. ${ }^{46}$

Mit der Kritik und Infragestellung der Schulkapitelstrukturen wurden die eigenen routinisierten Handlungsabläufe auf einmal ausdrücklich gemacht und neu bewertet. Die Irritation der alltäglichen Routine mobilisierte praktisches Wissen. ${ }^{47}$ Emil Jucker repräsentierte in dieser Praxis einen die Strukturen „bewusstmachenden Kritiker", der streckenweise gegen Konformitätszwänge opponierte. Derartige bewusstmachende Kritik operierte im Modus einer Institutionenkritik, die bei den gesellschaftlichen Institutionen ansetzte und das Ausmaß der in ihr eingelassenen strukturellen Gewalt zum Vorschein brachte, aber gleichzeitig Vertrauen in das Potenzial des Lehrpersonengremiums hatte. So zumindest ließe sich die bereits erwähnte Formulierung verstehen, dass Jucker die jetzige Organisation der Sektionen und Schulkapitel nicht gefalle. Der Beschluss von 1913 wurde interessanterweise zunächst nicht in Kraft gesetzt, es kam zu keiner Veränderung der gewohnten Praxis, andere kriegsbedingte Themen drängten sich vor. Erst im Mai 1918 geriet die Frage nach dem Fortbestand oder die Aufhebung der Sektionen wieder stark in den Blick des Schulkapitels. Emil Jucker referierte:

Die Sektionen in ihrer bisherigen Form haben sich überlebt. Es soll ein Provisorium nach neuen Grundsätzen geschaffen werden. Der Sprechende wünscht Arbeitsgemeinschaften und zwar in dem Sinne, dass die Arbeit von Lehrerschaft und Schule auch nach aussen hin zur Geltung kommt, - keine passiven Redekränzchen. ${ }^{48}$

Mit dieser Äußerung deutet sich an, dass die Zeit des Ersten Weltkrieges als gesamteuropäisches Phänomen eine Zäsur markieren und als Katalysator wirken konnte. ${ }^{49}$ Eine Transformation der Schulkapitel stand bevor. Juckers Rede von den Sektionen, die sich in ihrer bisherigen Form überlebt hätten, sollte wohl den Anschein von einem naturwüchsigen und unabänderlichen Prozess des Wandels von Sektionen geben. Doch dieses Verständnis teilten offenbar nicht alle Kapitelteilnehmende. Lehrer Huber aus Rüti formulierte einen Gegenantrag: „Die Sektionen möchten in dem bisherigen Sinne wenigstens solange bestehen, bis die Frage der Auflösungen in den obligatorischen Sektionsversammlungen geprüft worden sei“ ${ }^{\prime} .{ }^{50}$ Zwar versuchten Anhänger von Jucker, wie etwa Lehrer Dubs aus Hinwil, einen weiteren Antrag mehrheitsfähig

\footnotetext{
${ }^{46}$ Schulkapitel Hinwil, 24.05.1913, FBP, ZH HC III 4, 5, S. 268.

${ }^{47}$ Vgl. Jaeggi 2014, S. 12.

${ }^{48}$ Schulkapitel Hinwil, 11.05.1918, FBP, ZH HC III 4, 5, S. 351.

${ }^{49}$ Vgl. Barth 2016, S. 154f.

${ }^{50}$ Schulkapitel Hinwil, 11.05.1918, FBP, ZH HC III 4, 5, S. 351.
} 
werden zu lassen, demzufolge die Sektionen sofort aufgehoben ,und an ihrer Stelle kleine beweglich Apparate, nämlich Arbeitsgemeinschaften [...] auf freiwilliger Basis [treten]" sollten, aber in der Abstimmung wurde der Antrag Huber angenommen..$^{51}$ Dubs Antrag wäre bei einem Erfolg auf eine Erprobung neuer Repräsentationsformen hinausgelaufen. Die Vorstellung einer Wahl von kleinen Basiseinheiten durch die mitwirkenden Mitglieder, wie sie Jucker und Dubs mit den Arbeitsgemeinschaften vorschlugen, erscheint aus der Retrospektive nicht unberührt von damals zirkulierenden rätedemokratischen Modellen gewesen zu sein. ${ }^{52}$ Zumindest, so hielt es 1933 Emil Gassmann fest, dachte in der Zeit nach dem Ersten Weltkrieg

die überwiegende Zahl der Volksschullehrer nicht [...] an eine vollständige Beseitigung der Kapitelversammlungen; denn mit der Organisation der Kapitel ist das wichtige Recht verbunden, Abgeordnete in die Schulaufsichtsbehörde, die Bezirksschulpflege zu wählen. ${ }^{53}$

Ziemlich genau ein Jahr später fand die bildungspolitisch aufgeladene Debatte im Mai 1919 im Wirtshaus „Schweizerhof“ ihren prägnanten Ausdruck in einer Versammlung des Schulkapitels. ${ }^{54}$ Die Betriebstemperatur durch intensive Debatten schien sich 1919 ohnehin stark erhöht zu haben: „Da die Diskussion voll in Anspruch genommen werden dürfte, [...] wird [...] um pünktliches Erscheinen gleich nach Eintreffen der Züge gebeten“, hieß es auf der Einladung zur gemeinsamen Versammlung der Schulkapitel Hinwil, Pfäffikon und Uster. Anstelle des verhinderten Sekundarlehrers Huber referierte Lehrer Binder. Er versuchte - obwohl Huber Skepsis gegenüber dem Vorgehen der Fraktion Juckers, die Sektionen sofort abzuschaffen, geweckt hatte -, die Auflösung der Sektionen zu fundieren und für die Einführung von freien Vereinigungen auf der Grundlage eines Arbeitsprogramms zu plädieren. ${ }^{55}$

So haben die Sektionen nicht das gebracht, was durch ihre Gründer von ihnen erwartet wurde. Wohl seien manche gediegene Vorträge gehalten und ausführlich Programme aufgestellt worden, aber es sei nicht gelungen, die Mitglieder zu „mitarbeitenden Mitgliedern heranzuziehen“, führte der Referent Binder aus. Die meisten beschränkten sich auf das Zuhören und einige Wenige arbeiteten. Der Grund für diesen Misserfolg sei in den Zeitumständen zu suchen: Lehrer verbrächten viele Monate im Militärdienst und durch ungünstige

\footnotetext{
${ }^{51}$ Ebd., S. 352.

${ }^{52}$ Vgl. Förster 2018, S. 31.

53 Gassmann 1933, S. 616.

${ }^{54}$ Vgl. Schulkapitel Hinwil, 06.09.1919, FBP, ZH HC III 4, S. 5.

55 Ebd.
} 
Verkehrsverhältnisse seien Mitglieder nur schwer zu Versammlungen zusammenzubringen. ${ }^{56}$ Die widerspruchslose Annahme des Antrags, die Sektionen aufzulösen, wurde bemerkenswerterweise mit dem für Pädagogen wohl schmerzhaften Scheitern in Verbindung gebracht, die Mitglieder zu erziehen. Aber auch das Format der freien und selbstorganisierten Vereinigungen setzte auf Pädagogisierung: Diese sollte jedoch, ähnlich wie bei bürgerlichen Selbstbildungskonzepten, bereits weitgehend abgeschlossen sein, so dass sich gleichgesinnte Erwachsene engagiert und frei austauschen können. Es ist nicht auszuschließen, dass die Krisen- und Kriegszeit vor und während des Ersten Weltkriegs einerseits den institutionellen Veränderungsdruck förderte, jedoch andererseits - als eine Art weitere Bedeutungsschicht - die Pädagogisierungsambitionen dem „Austesten von Grenzen des Protests“ zuwiderliefen. ${ }^{57}$ Die Auflösung der Sektionen bedeutete eine Zäsur, waren doch die Schulkapitel und ihre Sektionen eine mit der Entfaltung der öffentlichen Volksschule im 19. Jahrhundert anerkannte und in Sachen Lehrpersonenfortbildung erfolgreiche Institution, die bildungspolitische Prozesse eines Wissensaufbaus initiierte und begleitete. Die mit ihr verbundene spezielle Form der Wissensproduktion durch selbstorganisierte Referate seitens der Kapitelmitglieder oder externer Referenten aus den Bereichen der Wissenschaft, Politik, Wirtschaft und Medizin gehörte wie ein Schatten zu der ständigen Arbeit des Gremiums. Die reflexiven Formen der verhandelten verschiedenen Wissen bedingten mit, dass die Schulkapitel, wie in Hinwil, sich immer wieder selbst thematisierten, kritisierten und reformierten - eben auch, wenn die Erziehungsambitionen gegenüber den eigenen Mitgliedern fehlschlugen.

\section{Wissens- und ideologische Deutungskämpfe}

Die Mitglieder der Schulkapitel verfolgten eine Art Erkenntnismodell der pädagogischen Beobachtung: Die Leitfäden der Lehrpersonenbildung wurden als unwissenschaftlich entlarvt und Wissensbestände und Vermittlungssettings in Frage gestellt. In der intensiv geführten Debatte, wo die Grenzen des erziehenden Unterrichts liegen könnten, wurden mehrheitlich Vorbehalte gegen eine das Lehrerhandeln einschränkende Perspektive geäußert. Während dieser Kontroverse veränderten sich die Praktiken der Dokumentation.

\footnotetext{
${ }^{56}$ Ebd.

${ }^{57}$ Vgl. Tanner 2014, S. 15.
} 


\subsection{Lehrerseminar, schulisches Wissen und Dokumentationspraktiken}

Die Selbstreflexion und Infragestellung der Schulkapitel als Weiterbildungsinstitution wurde flankiert durch das Simulieren eines quasi-akademischen Habitus. Dementsprechend wurden von den Lehrpersonen nicht nur die institutionalisierten Formen der Schulkapitel mit ihren Sektionen hinterfragt, sondern auch Lehrerseminare als Orte, die eine eher schulische Wissensproduktion organisierten: „Die heutige Lehrerbildung riecht an allen Ecken und Enden nach dem Leitfaden" - diese Zuschreibung des Sekundarlehrers Rudolf Brunner an der Kapitelversammlung im Bezirk Affoltern im Mai 1918 zielte auf die zeitgenössische Wahrnehmung, dass es der Lehrerbildung nicht möglich sei, aus unreifen Personen starke freie Menschen zu machen und mündete in die Frage: „Wie lange soll noch dem werdenden Lehrer die Hochschule verschlossen bleiben?" ${ }^{58}$ Sowohl das vier- als auch das fünfklassige Seminar wurden als berufliche Sackgasse abgelehnt. Die Berufsbildung sollte gemäß den Forderungen von Seiten der organisierten Lehrpersonen an der Hochschule stattfinden und von der allgemeinbildenden Mittelschule abgetrennt sein. Damit lag eine Positionierung der Unterrichtenden zu Beginn des 20. Jahrhunderts vor, die im Kanton Zürich nach dem Scheitern 1872 des Unterrichtsgesetzes des Erziehungsdirektors Johann Kaspar Sieber, welches eine Verlegung der Lehrpersonenbildung an die Universität verlangte, erst einmal nicht mehr unterstützt wurde. Unter der Leitung des Erziehungsdirektors Heinrich Mousson (Amtszeit: 1914-1929) wurde die Primarlehrerausbildung in Seminaren und eine Lehrerbildungsreform der kleinen Schritte gutgeheißen. ${ }^{59}$

Die Rhetorik der verhassten Leitfaden-Lehrerbildung lag vielen bildungspolitischen Exponenten der organisierten Lehrpersonen auf der Zunge. Sie fürchteten, dass unter den Bedingungen der anlaufenden sozialtechnologisch inspirierten Moderne viele Berufe außerhalb des Schulfelds akademisiert wurden, während die Lehrpersonenbildung in der eigenen Wahrnehmung am Seminar in einem Zustand aus pädagogischen Schlagwörtern und Maximen verbleibt, die sich in formelhaften Lehrbuchwissen erschöpften: ${ }^{60}$ „Da macht sich Schablonismus breit, ein handwerkmäßiges Arbeiten nach bewährter ,Methode', geheiligt durch jahrelange Verwendung und geschätzt vor allem wegen - ihrer Bequemlichkeit“, so Konrad Brandenberger 1918 in der Schweizerischen Lehrerzeitung (SLZ) in einem Beitrag mit dem Titel Lehrer und pädagogische Wissenschaften. ${ }^{61}$ Die Diskussionen und Forderungen zu einer Verlegung der

\footnotetext{
${ }^{58}$ Schulkapitel Affoltern, 11.05.1918, FBP, ZH HC III 1, S. 6.

${ }^{59}$ Vgl. Lussi Borer/Criblez 2011, S. 254; Ziegler 1998, S. 35.

${ }^{60}$ Vgl. Gugerli/Kupper/Speich 2005, S. 19.

${ }^{61}$ Brandenberger 1918, S. 148.
} 
gesamten Lehrpersonenbildung an die Hochschule waren im Kanton Zürich nicht neu, sondern begleiteten sie seit diese staatlich organisiert wurde. ${ }^{62}$ Aus einer praxeologisch sensiblen Sicht fällt auf, dass die Art der Dokumentation und Protokollierung im Schulkapitel sich veränderte. Ein Umbruch in der Dokumentationsform lässt sich für das Jahr 1918 ermitteln: Zuvor wurden die Sitzungen handschriftlich protokolliert und lediglich zu ausgewählten Versammlungen klebten die Schreibenden in das Protokollbuch an den Anfang der Aufzeichnungen die gedruckte Einladung hinein. Mit dem Jahr 1918 ist zu beobachten, dass nicht nur für besondere Versammlungen, sondern auch für als bedeutsam geltende Vorträge Ankündigungen oder Forderungen auf Handzetteln resp. Flugblättern gedruckt wurden, die dann - so die Annahme - in das handschriftliche Protokoll integriert werden sollten. Diese neue Instrumentierung verlieh dem Protokoll eine Art performative Bedeutung: ${ }^{63}$ Flugblätter wurden nicht nur Teil des Protokolls, sondern zuvor gleichzeitig zum Anlass und zentralen Gegenstand von Debatte und Kommentar. Die gedruckten Leitsätze der Lehrerbildungskommission des Schulkapitels Hinwil könnten einen Hinweis für die bildungspolitische Identifikation vieler Mitglieder mit den Akademisierungs- und Verwissenschaftlichungsforderungen geben. Nach dem Willen der meisten Mitglieder sollte den Forderungen mit einer Trennung der Lehrpersonenbildung in eine vorbereitende, allgemeinbildende - und allen Mädchen offenstehende - Mittelschule und in eine universitäre mindestens dreisemestrige Berufsausbildung begegnet werden. Für eine solche hochschulmäßige Gestalt galten die Auflösungen des kantonalen Seminars Küsnacht und der Seminarabteilung der städtischen höheren Töchterschule bzw. ihre Umwandlung in neusprachliche Gymnasien als notwendig. ${ }^{64}$

Die Leitsätze erfassten den Erwartungshorizont vieler Kapitelteilnehmenden und erinnern heute daran, dass eine Praxis in diesem Gremium darin bestand, in Alternativen und Optionen zu denken - was wiederum ein Kennzeichen für „,neue Unsicherheiten und neue Möglichkeiten“ ${ }^{65}$ generell für die Offenheit der damaligen Lehrerbildungssituation ist. In der Praxis liefen die Forderungen gegenüber einer universitären Umgestaltung auf Anliegen hinaus, die nicht nur an verschiedenen Orten der Lehrpersonenbildung in der Luft lagen, sondern an Seminaren durch einzelne Gruppierungen oder Akteure als Versuchsanordnungen, auf Probebühnen und Umschlagplätzen für neue Wissensordnungen bereits Konkretisierungen erfahren hatten. Denn die Verknüpfung von wissenschaftlichen und pädagogisch-schulpraktischen Wissen war bereits in vollem

\footnotetext{
${ }^{62}$ Grube/Hoffmann-Ocon 2015, S. 40f.

${ }^{63} \mathrm{Vgl}$. Hess 2015, S. 85.

${ }^{64}$ Hoffmann-Ocon 2016b, S. $163 f$.

${ }^{65}$ Vgl. Müller/Tooze 2015, S. 10.
} 
Gang: Wissenschaftlichkeit in der Lehrpersonenbildung konnte auch ein Ensemble von Tugenden, Werten und Haltungen bedeuten, das die Unterrichtspraxis anleiten und steuern sollte, ${ }^{66}$ so wie es in Übungsschule an der Universität Zürich realisiert wurde, in der sowohl seminaristisch als auch universitär Studierende Unterrichtspraktika absolvierten. ${ }^{67}$ Weiterführend könnte gefragt werden, ob im Zürcher Fall hinter der Idee der Universität als einziger Ort der Lehrpersonenbildung ein eigenständiger Diskurs über die Akademisierung des Lehrberufs stand oder ob diese Forderungen einer Kritik von etablierten Seminarorten folgten.

\subsection{Pädagogische Lebenslügen und Grenzen der Erziehung}

Der Ton in den Protokollaufzeichnungen der Schulkapitel ist in der Retrospektive sehr unterschiedlich - teilweise wirkt er nüchtern-distanziert, gerade wenn es um Zusammenfassungen einzelner Vorträge ging, teilweise wirkt er aktivistisch, wenn von Abstimmungen berichtet wurde. Die Debatten nach sich ziehenden Referate basierten oftmals auf der zeitgenössischen Vielfalt an politischen, philosophischen und ökonomischen Ideen. Das Debattenklima unter den Kapitelteilnehmenden bestand weniger aus einem „Vergangenheitsweh“, sondern drängte eher nach der Auflösung von pädagogischen „Lebenslügen““ ${ }^{68}$ Die versammelten Lehrpersonen beanspruchten die Auseinandersetzung mit diversen Gesellschafts- und Kulturkonzeptionen. Es kam in den Debatten zu Übernahmen, Kombinationen, Reduktionen und Verflechtungen einzelner Versatzstücke, die oftmals für das Berufs- und Schulfeld ,umgewertet“ wurden, so dass sich womöglich auch ein neuer Wissenstyp herausbildete.

Die Krisenzeit während und nach dem Ersten Weltkrieg hatte - zunächst nur bei einem kleineren Teil, der womöglich die Vergemeinschaftungs- und auch gleichzeitige Exklusionstendenzen durch Programmatiken der nationalen Erziehung skeptisch betrachtete -, ${ }^{69}$ Argwohn gegenüber umfassenden Pädagogisierungsansprüchen geweckt. Daher spielte das Thema „Grenzen der Erziehung “ eine entscheidende Rolle für die Diagnose, dass in einer dekadenten Zeit jeder jeden erziehen wolle: ${ }^{70}$ Dieses Urteil sickerte bis in die Schulkapitel hinein. In der im Schulhaus Aeugst im September 1921 stattfindenden Versammlung des Bezirks Affoltern reicherte Lehrer R. Bosshard aus Herferswil seinen Vortrag mit der Perspektive an, dass ein guter Erzieher nicht beabsichtige, durch seinen Willen auf anderen Menschen einzuwirken. Was Erzieher

\footnotetext{
${ }^{66}$ Vgl. Klein 2016, S. 139.

67 Vgl. Stettbacher 1916.

68 Vgl. Strasser 2018, S. 22.

${ }^{69}$ Vgl. Hoffmann-Ocon/Bascio 2018.

${ }^{70}$ Vgl. Grisebach 1924, S. $7 f$.
} 
vielmehr könnten sei, diese negativen Kräfte zu bekämpfen. Unterrichtsmethoden seien Umwege: „Hätte der grössere Teil der Lehrerschaft so gewirkt, heute stünde es um die Menschheit anders“ ${ }^{71}{ }^{71}$ Hier standen die überspannten Erziehungsbemühungen zu einer Gemeinschaft während der Kriegs- und Krisenzeit in der Kritik. Bosshard führte weiter aus, dass der Lehrer in jedem Schüler ein Individuum sehen müsse, dessen Entwicklung er nicht hemmen, sondern nur leiten solle. Die Nivellierung sei der Feind der Persönlichkeitsbildung, der Untergang alles Großen. Psychologisches Studium täte mehr not als das Arbeitsprinzip. ${ }^{72}$ So wandte er sich gegen Positionen, wie diejenige des unter Zürchern Pädagogen hoch gehandelten Arbeitsschulpädagogen, Sozialdemokraten und Grütlianers Robert Seidel, der noch 1915 vor dem Schulkapitel Zürich im Tonhalle-Pavillon für ein Ende der Individualpädagogik plädiert und nach einer Erziehung des „Menschen für den Staat“ und als „Glied der Volksgemeinschaft" gerufen hatte. ${ }^{73}$

Der Lehrer, der die an sich individuell begabten und talentierten Schüler zur Mediokrität drängt, sie in den Worten von Bosshard ,nivelliert“, eben seine Grenzen des Erziehungsgeschehens nicht erkennt und Individualität zerstört, war der Stoff zeitgenössischer Bildungs- und Schulkritik. Insbesondere für anti-egalitäre Konnotationen und Versatzstücke wurden die Werke Friedrich Nietzsches als Fundgrube genutzt, aus der sich von der Argumentationsbildung her auch die Mitglieder der Schulkapitel bedienten. ${ }^{74}$ In seinen Basler Vorlesungen, die unter dem Titel Über die Zukunft unserer Bildungsanstalten (1872) später publiziert wurden, äußerte Nietzsche: „Nicht Bildung der Masse kann unser Ziel sein: sondern Bildung der einzelnen ausgelesenen, für große und bleibende Werke ausgerüsteten Menschen“. ${ }^{75}$ Für Volksschullehrpersonen mussten seine effektvollen Thesen eigentlich ein Greuel sein. Sie waren dazu angetan, elitäre Implikationen hervorzurufen. Dennoch blieb die Lektüre seiner Bücher sowohl unter Seminarlehrpersonen als auch Seminaristen populär. Der Gründungsdirektor des Zürcher Oberseminars, Walter Guyer, sprach etwa in seinem 1949 an die Seminarkandidaten adressierten Lehrbuch rückblickend davon, dass in den

\footnotetext{
${ }^{71}$ Schulkapitel Affoltern, 17.09.1921, FBP, ZH HC III 1, S. 6.

${ }^{72}$ Vgl. Schulkapitel Affoltern, 17.09.1921, FBP, ZH HC III 1, S. 6.

${ }^{73}$ Schulkapitel Zürich, Abteil I-IV, 27.02.1915; Hoffmann-Ocon 2016, S. 257a.

${ }^{74}$ Vgl. Niemeyer 2016, S. 54.

75 Nietzsche 1912, S. 333.
} 
letzten vierzig Jahren $[\ldots]$ Nietzsches aufrüttelnde $[\ldots]$ Gedanken $[\ldots]$ nicht nur zu einer realistischen Sicht in die Abgründe der menschlichen Existenz, sondern oft auch nur zur eigentlichen Verzweiflung und zur Kapitulation vor der Entscheidung

führten. ${ }^{76}$ Womöglich kam es aber auch zu einer differenzierten Wahrnehmung durch die Volksschullehrpersonen, die sich mit Nietzsches Aussagen über die zunehmende Gleichgültigkeit der Jugend gegenüber nicht unmittelbar verwertbaren Wissensbeständen stark identifizieren konnten. Nietzsche zielte auf eine wahrgenommene Bildungsströmung ab, in der es als vordringlichste Aufgabe erscheint, ,,couranten“ Menschen eine rasche Bildung zu vermitteln, damit diese schnell zu geldverdienenden Wesen werden könnten. ${ }^{77}$ Selbst der in Zürich Aussersihl tätige Arbeiterarzt, Sozialdemokrat und Anarchist Fritz Brupbacher thematisierte anhand der Nietzsche-Lektüre die Befreiung des Individuums und die Revolte gegen die Bourgeoisie. ${ }^{78}$

Während die Werke von Nietzsche bereits jahrzehntelang vorlagen, jedoch erst an der Schwelle zum 20. Jahrhunderts zum populären Gegenstand der Reflexion wurden, legte der Philosoph und Pädagoge Eberhard Grisebach seinen direkt nach dem Ersten Weltkrieg publizierten Überlegungen die Annahme der Grenzen der Erziehung zugrunde. Grisebachs Ansatz - der „Bildung“ nur als „Selbstbildung“ gelten ließ, als ein Wissen um eigene Grenzen, die uns den „Abstand von der Vollendung“" zeigen - vertrug sich nicht mit der Pädagogik im Sinne volkserzieherischen Denkens. ${ }^{79}$ Demnach widerspricht „Gebildetsein“ dem Wesen der Bildung, Bildung sei nie vollendet und erfordere „Fragen" und „ewiges Forschen“. ${ }^{80}$ „Jeder will heute jeden erziehen“, diese bereits erwähnte und von Grisebach als „Erziehungssucht“ etikettierte Verhalten, dieses Durcheinanderschreien von verschiedenen „Ich-Parteien“, die mit ihrem Willen auf andere einwirken wollen, ganz ähnlich wie Bosshard es vor dem Schulkapitel referierte, war für Grisebach ein Ausdruck von Anarchie und Dekadenz der unmittelbaren Nachkriegszeit. ${ }^{81}$

\subsection{Die pädagogische Revolution}

Wenn man nach Referenzen und Kontexten der Vorträge sucht, die während der Schulkapitelversammlungen präsentiert wurden, wird die intensive Beschäftigung mit damals aktuellen, pädagogisch relevanten Schriften besonders

\footnotetext{
${ }^{76}$ Guyer 1949, S. 290.

${ }^{77}$ Nietzsche 1912, S. 298 f.

${ }^{78}$ Vgl. Michalzik 2018, S. 43.

${ }^{79}$ Grisebach 1919, S. 375-377.

${ }^{80}$ Ebd., S. 377.

${ }^{81}$ Grisebach 1924, S. 38.
} 
deutlich. Eine Vorliebe für Schriften mit pathetischer Tonlage und bildungspolitischer Programmatik ist dabei zu beobachten. Allerdings ließen sich die Lehrpersonen von scheinbar neuen Betrachtungsweisen nicht automatisch dominieren, sondern verstärkten - fast schon in ritueller Weise - ihre Bemühungen, diese mit dem Berufs- und Schulfeldbezug vorherrschende pädagogische Ordnungen zur Geltung zu bringen.

Dem Protokoll der Schulkapitelversammlung von Horgen Anfang Oktober 1920 zufolge erstreckte sich der Vortrag des Sekundarlehrers J. Eugster aus Wädenswil im Schulhaus Samstagern (heute Gemeinde Richterswil) ohne Pause auf volle eineinhalb Stunden. Gefiltert durch seine Lektüre stellte er Leonhard Ragaz‘ Überlegungen zur Pädagogischen Revolution vor:

Pfarrer Ragaz will den Aufbau der Zukunftsschule in Form einer religiösen, sozialen und pädagogischen Revolution durchführen. Er ist kein Freund der Staatsschule, obschon er ja auch Staatsbrot isst. Das rührt wohl von seiner ganz sonderbaren Staatsauffassung her, bei der kein geordneter Staat bestehen könnte. ${ }^{82}$

Ragaz, der Pfarrer mit dem Sensorium für soziale Anliegen, trat zu Beginn der 1920er-Jahre, also zur Zeit der Publikation der Pädagogischen Revolution, von seiner Professur für systematische und praktische Theologie an der Universität Zürich zurück, um eine religiös-soziale Bewegung mit Tätigkeitsfeld im Zürcher Arbeiterquartier Aussersihl zu befeuern. ${ }^{83}$ Seine Entwürfe für Hochschule und Volksschule verstand er als Ideal, als ein „Programm für eine lange Arbeit und einen grossen Kampf" und ,nicht [als] etwas, das morgen oder übermorgen durch eine Behörde oder ein Komitee gemacht werden kann““ ${ }^{84}$ Diese langfristige und auf eine Art soziale Erlösung zielende Anlage schien in der Schulkapitelversammlung wenig gewürdigt worden zu sein, auch wenn der Erlösungsgedanke in Bezug auf die Grippewelle noch verfangen hatte. Die Empörung bezog sich vor allem auf den antistaatlichen Charakter der Schulideen und der Schulkritik des Armenpfarrers. Dazu bemerkt das Protokoll:

Ragaz verlangt freie Schulgemeinden, nach religiösen, politischen und sozialen Gesichtspunkten. Ein ganz verführerisches Zukunftsbild entwirft Ragaz von der Freiheit dieser neuen Schule. Seine Losung ist: Weg mit dem Schulhaus, Lehrplan, Stundenplan, Schulmeister und Schulmeisterstock und all dem Kasernengraus. Auch der Schulzwang fällt weg [...]. Mit grossem Geschick verteidigt sich Herr Eugster gegen die Angriffe von Herrn Ragaz. Mit Recht verweist er ihn auf sein Arbeitsfeld. Auf

\footnotetext{
${ }^{82}$ Schulkapitel Horgen, 02.10.1920, FBP, ZH HC III, 2, 2 a.

${ }^{83}$ Vgl. Brassel-Moser 2012.

${ }^{84}$ Ragaz 1920, S. 102.
} 
kirchlich-religiösem Gebiete könnte er viel Fruchtbares schaffen als an der Institution unserer Schule herum zu nörgeln. ${ }^{85}$

Ragaz' Hoffnung zielte darauf, jegliche Form von Standesschule mit zwangsmäßigen und staatsdienenden Charakter zu beseitigen; ${ }^{86}$ alle Schülerinnen und Schüler sollten die Mittelschule besuchen können, welche soziale Gerechtigkeit fördere. Schulen wurden dabei als demokratisch-öffentliche Räume gedacht, die auf lokaler Ebene durch die Praxis der Lehrpersonen und gesteuert durch Rückbindung an Vor-Ort-Gremien wirken. ${ }^{87}$ Die nach Bezirken organisierten Schulkapitel hätten womöglich diese Funktionen einnehmen können. Aber die effektvollen Zukunftsbilder von Ragaz wurden mit erkenntniskritischen Bremsmanövern unter dem Hinweis der Verführung abgelehnt.

Die Mehrheit der Schulkapitelteilnehmenden schien Ragaz‘ Zukunftsentwürfen kurz nach dem Ersten Weltkrieg eher zu misstrauen - getreu dem Motto: „Träume vom Paradies führen in die Hölle! “88 Mit dem Vielerlei an bildungspolitischen Zukünften und divergierenden programmatischen Vorstellungen zeigte sich im Mikrokosmos der Schulkapitel, was Lucian Hölscher als die „längerfristigen Grundkonflikte“ und „,inneren Spannungen“ bezeichnet, welche fast alle europäischen Gesellschaften im Zuge des industriellen Transformationsprozesses um 1900 erfasst hatten und diese, ,in sozial und weltanschaulich stark divergierende Lager zerfallen“ ließen. ${ }^{89}$ Die soziale Revolutionsperspektive wurde zum Fluchtpunkt von positiven wie negativen Erfahrungen mit Zukunftsentwürfen einzelner Akteure. Während die im weiteren Sinne sozialistisch Inspirierten mit ihren bildungspolitischen Alltagspraktiken und gemeinsamen Deutungen darauf sannen, dass Kommen der sozialen Revolution in der Hoffnung auf eine gesellschaftliche Erlösung zu beschleunigen, versuchten die Revolutionsgegner dies teilweise mit „Gegenrevolutionen“ oder konservativen Wenden zu verhindern oder in ständestaatliche und autokratische Regime umzumünzen. Insbesondere Ragaz erzeugte mit der Verknüpfung von religiösen, politischen und sozialen Gesichtspunkten in Form der pädagogischen Revolution einen eschatologischen Horizont, der auch als mentaler Schutz gegenüber den sozialen Leidenserfahrungen Vieler kurz nach der Kriegszeit gedeutet werden kann. Nicht zwingend als Ausdruck für eine Erosion von tradierten gesellschaftlichen Milieus, aber als Merkmal für einen fortgeschrittenen „Verbürgerlichungsprozess“ der Volksschullehrpersonen könn-

\footnotetext{
${ }^{85}$ Schulkapitel Horgen, 02.10.1920, FBP, ZH HC III, 2, 2a.

${ }^{86}$ Ragaz 1920, S. 104.

${ }^{87}$ Vgl. Gabbard 2009, S. 28.

${ }^{88}$ Radkau 2017, S. 15.

${ }^{89}$ Hölscher 2016, S. 241.
} 
ten die Abwehrreaktionen der Horgener Schulkapitelteilnehmenden verstanden werden. Die Revolutionsfurcht in diesem Publikum, das sich für gesellschaftliche und moderate Schulreformen durchaus mobilisieren ließ, schien erheblich gewesen sein; da nahm man soziale Unerlöstheit in Kauf und ersetzte die politische revolutionäre Überschreitung mit Überschreiten im Denken, etwa indem das vorherrschende Bildungssystem irritierende Ansätze referiert und damit im Raum bildungspolitischer Realitäten gleichsam domestiziert wurden.

\section{Resümee}

In den ersten Jahren nach dem Ersten Weltkrieg strukturierten neben entscheidenden Demokratisierungsschüben neue Unsicherheiten und neue Möglichkeiten den bildungspolitischen Handlungshorizont. ${ }^{90}$ Erweiterungen im Wahlrecht standen vielerorts zur Debatte und waren Element von ungleichen, aber gleichzeitigen Entwicklungen resp. Demokratisierungsepisoden. In den Schulkapiteln kam es in einzelnen Bezirken zu enormen bildungspolitischen „Geschwindigkeitssteigerungen" bis hin zur Abschaffung tradierter Gremienformen. Die Protagonisten in den Schulkapiteln oszillierten, womöglich aus Angst vor einem nichtgeordneten Ausnahmezustand, sowohl zwischen den Polen revolutionärer Rhetorik und Tradierung der Gremienlogiken des Politischen als auch zwischen schulischen Gremien und außerschulischen politischen Verbänden. Die Schulkapitel blieben nach 1918 bestehen, allerdings wurden die Binnenstruktur durch die Auflösung der Sektionen verändert. Eine Begleiterscheinung war, dass die Schulkapitel nicht mehr exklusive Orte der bildungspolitischen Meinungsbildung waren und deutlicher den Charakter eines Fortbildungsgremiums bekamen.

Die noch weiter zu erforschende Geschichte der Schulkapitel lässt sich mit einer moderat praxeologischen Perspektive als Geschichte multipler Temporalitäten nachspüren. Fraglich bleibt, ob sich die Jahre zwischen 1917 und 1923 für die Untersuchung der Schulkapitel als eigene Epoche zusammenfassen lassen, wie Daniel Schönpflug dies mit dem Begriff der Kometenjahre vorschlägt. ${ }^{91}$ Auf eine Sehnsucht nach neuen Formaten der Auseinandersetzung deutet einiges hin. Der Wunsch nach Veränderung der Fortbildungspraxis erzeugte eine breit abgestützte bildungspolitische Diskussionskultur und eine gewisse organisationale Experimentierfreudigkeit, allerdings schien die gerade hinter sich gelassene verstörende Gegenwart auch wenig Lust auf gefährlich

\footnotetext{
${ }^{90}$ Müller/Tooze 2015, S. 10.

${ }^{91}$ Schönpflug 2017, S. 307.
} 
erscheinende Zukunftsentwürfe gemacht zu haben. Mögliche Thesen von den Schulkapiteln als Kreativlabor erscheinen daher überzogen und würden verkennen, dass tatsächlich revolutionäre Themen in einer Gremienpraxis und mit einem Veränderungen eher fürchtenden Schulfeld und -personal auch ausgekühlt werden konnten. Die Analyse materieller Überlieferung, hier vor allem die Protokolle der Schulkapitelversammlungen, zeigt mit Blick auf Umgangsweisen in Gremien und Gebrauchsweisen von Ankündigungen und Flugschriften deutliche Verschiebungen. Eine solche Dokumentationspraxis verweist auf eine weitere Bedeutungsebene. Neben administrativen, quasi-schulbehördlichen und proto-akademischen Aspekten eines Protokolls, die den Charakter der wissenschaftlichen Durchdringung des verhandelten Gegenstands nahelegen konnten, konnte die neue Dokumentationsform als eine berufsständische Strategie begriffen werden, sich jenseits der Simulation gelehrter Rituale einer bildungspolitischen Anerkennung zu versichern. ${ }^{92}$

Brüche, die auf grundsätzliche Veränderungen von Interpretationen geteilter Praktiken hinweisen, schienen eher selten gewesen zu sein..$^{93}$ Vielmehr lagen, „ein andauerndes (Wechsel-)Spiel zwischen Kontingenz und Kohärenz vergangener Alltagspraxis" vorausgesetzt, ${ }^{94}$ Irritationen von Routineschleifen in äußerlichen Ereignissen wie der in Grippewelle. Die Auflösung der Sektionen der Kapitelkonferenzen erfolgte wiederum als eine gemeinsam beschlossene Transformation des Bestehenden. Inwiefern dies auch die Praxis in einigen Schulbezirken veränderte, sich in Gastwirtschaften zu Versammlungen in einer Atmosphäre zu treffen, welche die politische Debatte befeuerte, wäre zukünftig weiter zu ermitteln. Womöglich war aus Sicht der Schulkapitelmitglieder ,ihr Motor [...] die Affirmation des Unbestimmten. Sie bejahte[n] das Bild einer offenen Zukunft, statt in idealistischen oder utopistischen Träumereien“ gefestigte bildungspolitische Wege zu verlassen. ${ }^{95}$

\section{Quellen- und Literaturverzeichnis}

\section{Ungedruckte Quellen}

\section{Forschungsbibliothek Pestalozzianum (FBP)}

Prot. Affoltern. Kapitelsversammlung, Samstag, den 11. Mai 1918, vormittags 9 Uhr im Schulhaus Hedingen, ZH HC III 1, 6.

Prot. Schulkapitel Hinwil 1902-1919. II. Kapitelsversammlung. Samstag, 24. Mai 1913 vormittags 9112 h im „Bären“ Bäretswil, ZH HC III 4, 5, S. 265-271.

\footnotetext{
${ }^{92}$ Hess 2015, S. 89.

${ }^{93}$ Jaeggi 2014, S. 106.

${ }_{94}$ Haasis/Rieske 2015a, S. 33.

${ }^{95}$ Steinweg 2018, S. 59.
} 
Prot. Schulkapitel Hinwil 1902-1919. III. Versammlung. Samstag, den 11. Mai 1918 im „Löwen“ Rüti, ZH HC III 4, 5, S. 350-353.

Prot. Schulkapitel Hinwil 1902-1919. Gemeinsame Veranstaltung Schulkapitel Hinwil, Pfäffikon und Uster. Sonntag den 6. September 1919 vormittags 91/2 Uhr im „Schweizerhof“ Wetzikon, ZH HC III 4, 5 .

Schulkapitel Horgen. Nekrologe. 3. Karl Sutter, Sek. Lehrer in Oberrieden (1893-1919). Nekrolog, gehalten von Herrn Rud. Wiederkehr in Oberrieden an der Kapitelsversammlung vom 29. Oktober 1919 in Horgen, ZH HC III 2, 1 b.

Protokoll der oberen Sektion des Kapitels Horgen. II. 1917-IV. 1929. Versammlung. Samstag, d. 30. November 1918 nachm. 2 Uhr im Sek-Schulhaus Richterswil, ZH HC III 2, 2a.

Protokoll der oberen Sektion des Kapitels Horgen. II. 1917-IV. 1929. Versammlung, Samstag, den 2. Oktober 1920 nachmittags 2 Uhr im Schulhaus Samstagern, ZH HC III 2, 2a.

Schulkapitel Zürich 1915. Abt. I - IV. 4. Abteilung. ASK 33. Versammlung. Samstag, den 27. Februar 1915, vormittags 83/4 Uhr im Tonhallepavillon Zürich (1915).

[Seidel, Robert]: Das Ziel der Erziehung vom Standpunkt der Sozialpädagogik. Vortrag von Herrn Nationalrat Robert Seidel, ZH HC III 19, 5.

Stettbacher, Hans (1916): Übungsschule 1909-16. Zürich.

\section{Schweizerisches Sozialarchiv (SOZARCH)}

Protokollbuch der Sozialdemokratischen Lehrervereinigung. 1906-1920: 1 Band; Versammlungsprotokolle 07.11.1906-21.05.1920 (Ar 201.252.1). Darin: Gründungssitzung vom 7. November 1906, S. 5-9; Versammlung. Mittwoch 10. Dez. [1906] ab[en]ds. 61/2 h im Schwanen Zürich; Ausserordentliche Versammlung vom 6. Oktober 1916, S. 222-224; Sitzung vom 2. September [1918] abends 5h, S. 267-269.

\section{Gedruckte Quellen}

Brandenberger, Konrad (1918): Lehrer und pädagogische Wissenschaften. In: Schweizerische Lehrerzeitung 63, H. 18, S. 147-148.

Gassmann, Emil (1933): Die zürcherische Volksschule und die ihr angegliederten Bildungs- und Wohlfahrtseinrichtungen von 1872 bis 1932: In: Erziehungsrat des Kanton Zürich (Hg.): Volksschule und Lehrerbildung 1832-1932. Zürich, S. 555-690.

Gassmann, Emil (1934): Zur Geschichte der zürcherischen Schulsynode. Beilage II. In: Kanton Zürich: Bericht über die Verhandlungen der Zürcherischen Schulsynode von 1934. Pfäffikon, S. 35-40.

Grisebach, Eberhard (1919): Die Wahrheit und Wirklichkeiten. Entwurf zu einem metaphysischen System. Halle/Saale.

Grisebach, Eberhard (1924): Die Grenzen des Erziehers und seine Verantwortung. Halle/Saale.

Guyer, Walter (1949): Grundlagen einer Erziehungs- und Bildungslehre. Zürich.

Keller, Emil (1934): 100 Jahre zürcherische Schulsynode. Beilage I. Kanton Zürich: Bericht über die Verhandlungen der Zürcherischen Schulsynode von 1934. Pfäffikon, S. 22-33.

Kreis, Hans (1933): Die zürcherische Volksschule von 1845 bis 1872. In: Erziehungsrat des Kanton Zürich (Hg.): Volksschule und Lehrerbildung 1832-1932. Zürich, S. 345-554.

Nietzsche, Friedrich (1912): Über die Zukunft unserer Bildungsanstalten. Aus dem Nachlass 1871/72. Leipzig.

Ragaz, Leonhard (1918): Die neue Schweiz. Ein Programm für Schweizer und solche, die es werden wollen. Olten.

Ragaz, Leonhard (1920): Die pädagogische Revolution. Zehn Vorlesungen zur Erneuerung der Kultur. Olten. 


\section{$132 \mid$ Andreas Hoffmann-Ocon}

\section{Literatur}

Barth, Boris (2016): Europa nach dem Grossen Krieg. Die Krise der Demokratie in der Zwischenkriegszeit 1918-1938. Frankfurt am Main.

Binotto, Johannes (2013): Tat/Ort. Das Unheimliche und sein Raum in der Kultur. Zürich/Berlin.

Brassel-Moser, Ruedi (2012): Leonhard Ragaz. In: Historisches Lexikon der Schweiz (HLS), Version vom 12.1.2010; https:/hls-dhs-dss.ch/de/articles/009059/2012-01-12/ [20.6.2019].

Buomberger, Thomas (2014): Kampfrhetorik, Revolutionsangst und Bürgerwehren. Der Landesstreik vom November 1918. In: Rossfeld, Roman/Buomberger, Thomas/Kury, Patrick (Hg.): 14/18. Die Schweiz und der Grosse Krieg. Baden, S. 336-365.

Bürgi, Markus (2010): Hans Oprecht. In: Historisches Lexikon der Schweiz (HLS), Version vom 16.9.2010; https://hls-dhs-dss.ch/de/articles/006464/2010-09-16/ [20.6.2019].

Desiderato, Simone/Lengwiler, Urs/Rothenbühler, Verena (2008): Jugendhilfe Kanton Zürich 1908-2008. Zwischen Professionalität und politischem Kräftemessen. Amt für Jugend und Berufsberatung (Hg.). Zürich.

Förster, Birte (2018): 1919: Ein Kontinent erfindet sich neu. Stuttgart.

Gabbard, David (2009): Anarchist Movement and Education. In: Ayers, William/Quinn, Therese/ Stovall, David (Hg.): Handbook of Social Justice in Education. New York, S. 24-29.

Graber, Rolf (2017): Demokratie und Revolten. Die Entstehung der direkten Demokratie in der Schweiz. Zürich.

Graf, Rüdiger (2008): Was macht die Theorie in der Geschichte? „Praxeologie“ als Anwendung des „gesunden Menschenverstandes“. In: Hacke, Jens/Pohlig, Matthias (Hg.): Theorie in der Geschichtswissenschaft. Einblicke in die Praxis des historischen Forschens. Frankfurt am Main, S. 109-129.

Grube, Norbert/Hoffmann-Ocon, Andreas (2015): Orte der Lehrerinnen- und Lehrerausbildung im Kanton Zürich - Überblick auf Dynamiken, Kontroversen und eine spannungsgeladene Vielfalt. In: Hoffmann-Ocon, Andreas (Hg.): Orte der Lehrerinnen- und Lehrerbildung im Kanton Zürich. Bern, S. 25-95.

Gugerli, David/Kupper, Patrick/Speich, Daniel (2005): Die Zukunftsmaschine. Konjunkturen der ETH Zürich 1855-2005. Zürich.

Haasis, Lucas/Rieske, Constantin (2015a): Historische Praxeologie. Zur Einführung. In: Hassis, Lucas/Rieske, Constantin (Hg.): Historische Praxeologie. Dimensionen vergangenen Handelns. Paderborn, S. 7-54.

Haasis, Lucas/Rieske, Constantin (2015b): Was ist und was kann die Historische Praxeologie? Ein runder Tisch. In: Haasis, Lucas/Rieske, Constantin (Hg.): Historische Praxeologie. Dimensionen vergangenen Handelns. Paderborn, S. 199-236.

Hess, Volker (2015): Schreiben als Praktik. In: Brendecke, Arndt (Hg.): Praktiken der frühen Neuzeit. Akteure - Handlungen - Artefakte. Köln, S. 82-99.

Hoffmann-Ocon, Andreas (2016a): Reflexion und Kritik des Ersten Weltkriegs in der Zürcher Schulsynode und den Schulkapiteln - Die Dynamik der Krisenwahrnehmung und der Wandel der individualpädagogischen Perspektive. In: Boser, Lukas/Bühler, Patrick/Hofmann, Michèle/ Müller, Philippe (Hg.): Pulverdampf und Kreidestaub. Beiträge zum Verhältnis zwischen Militär und Schule in der Schweiz im 19. und 20. Jahrhundert. Bern, S. 251-278.

Hoffmann-Ocon, Andreas (2016b): Das Fach Pädagogik in der Ausbildung von Volksschullehrpersonen zu Beginn des 20. Jahrhunderts. Bildungspolitische Deutungskämpfe im Kanton Zürich. In: Hoffmann-Ocon, Andreas/Horlacher, Rebekka (Hg.): Pädagogik und pädagogisches Wissen. Ambitionen in und Erwartungen an die Ausbildung von Lehrpersonen. Bad Heilbrunn, S. 151-170.

Hoffmann-Ocon, Andreas (2018): Der Streit um Konzeptionen im Feld der Lehrpersonenbildung. Reformdebatten in der Zürcher Schulsynode und in den Schulkapiteln vor 1938. In: Imlig, 
Flavian/Lehmann, Lukas/Manz, Karin (Hg.): Schule und Reform. Veränderungsabsichten, Wandel und Folgeprobleme. Wiesbaden, S. 52-64.

Hoffmann-Ocon, Andreas/Bascio, Tomas (2018): Nationale Erziehung als Kampffeld in der deutschsprachigen Schweiz während und nach dem Ersten Weltkrieg. In: Grube, Norbert/Hoffmann-Ocon, Andreas/De Vincenti, Andrea (Hg.): Erster Weltkrieg, Schule und Volksbildung in der Deutschschweiz. Pädagogisierungsambitionen und -dynamiken zwischen Mobilisierung und gesellschaftlicher Balance. Zürich, S. 167-199.

Hölscher, Lucian (2016): Die Entdeckung der Zukunft. Göttingen.

Jaeggi, Rahel (2014): Kritik von Lebensformen. Berlin.

Janz, Oliver (2013): 14. Der grosse Krieg. Frankfurt am Main/New York.

Klein, Ursula (2016): Nützliches Wissen. Die Erfindung der Technikwissenschaften. Göttingen.

Kraus, Katrin (2005): Zur berufspädagogischen Bedeutung des Betriebs als Lernort - oder: warum ein Krankenhaus kein Lernort im Sinne des berufspädagogischen Diskurses ist. In: Berufs- und Wirtschaftspädagogik - online, H. 9, S. 1-11.

http://www.bwpat.de/ausgabe9/kraus_bwpat9.shtml [20.6.2019].

Kreis, Georg (2014): Insel der unsicheren Geborgenheit. Die Schweiz in den Kriegsjahren 19141918. Zürich.

Lehmann, Johannes F./Thüring, Hubert (2015): Rettung und Erlösung. Einleitung. In: Lehmann, Johannes F./Thüring, Hubert (Hg.): Rettung und Erlösung. Politisches und religiöses Heil in der Moderne. Paderborn, S. 7-28.

Luhmann, Niklas (1996): Protest. Systemtheorie und soziale Bewegungen. Frankfurt am Main.

Lussi Borer, Valérie/Criblez, Lucien (2011): Die Formierung der Erziehungswissenschaften und die akademische Lehrerinnen- und Lehrerbildung. In: Hofstetter, Rita/Schneuwly, Bernard (Hg.): Zur Geschichte der Erziehungswissenschaften in der Schweiz. Vom Ende des 19. bis zur Mitte des 20. Jahrhunderts. Bern, S. 237-270.

Meier, Christian (2014): Der Historiker und der Zeitgenosse. Eine Zwischenbilanz. München.

Mersch, Dieter (2015): Epistemologien des Ästhetischen. Zürich.

Metz, Peter (2017): Mehrfachorientierung als Herausforderung für Eigenständigkeit - Zur Geschichte der SGL und ihrer Vorgängerverbände. In: Beiträge zur Lehrerinnen- und Lehrerbildung 35 , H. 0, S. 8-29.

Michalzik, Peter (2018): 1900. Vegetarier, Künstler und Visionäre suchen nach dem neuen Paradies. Köln.

Müller, Tim B./Tooze, Adam (2015): Demokratie nach dem Ersten Weltkrieg. In: Müller, Tim B./Tooze, Adam (Hg.): Normalität und Fragilität. Demokratie nach dem Ersten Weltkrieg. Hamburg, S. 9-36.

Niemeyer, Christian (2016): Nietzsche als Erzieher. Pädagogische Lektüren und Relektüren. Weinheim/Basel.

Nigro, Roberto (2015): Wahrheitsregime. Zürich/Berlin.

Radkau, Joachim (2017): Geschichte der Zukunft. Prognosen, Visionen, Irrungen in Deutschland von 1945 bis heute. München.

Raschke, Joachim (1985): Soziale Bewegungen. Ein historisch-systematischer Grundriss. Frankfurt am Main.

Schönpflug, Daniel (2017): Kometenjahre. 1918: Die Welt im Aufbruch. Frankfurt am Main.

Schroer, Markus (2005): Einleitung. Zur Soziologie des Körpers. In: Schroer, Markus (Hg.): Soziologie des Körpers. Frankfurt am Main, S. 7-47.

Senn, Martin A. (2018): Die Todeswelle. 25000 Menschen starben in der Schweiz 1918/19 an der Spanischen Grippe. In: NZZ Geschichte, Nr. 15, S. 39-49.

Sonderegger, Christian/Tscherrig, Andreas (2016): Die Grippepandemie 1918-1919 in der Schweiz. In: Krämer, Daniel/Pfister, Christian/Segesser, Daniel Marc (Hg.): „Woche für 


\section{4 | Andreas Hoffmann-Ocon}

Woche neue Preisaufschläge“. Nahrungsmittel-, Energie- und Ressourcenkonflikte in der Schweiz des Ersten Weltkrieges. Basel, S. 259-284.

Steinweg, Marcus (2018). Subjekt und Wahrheit. Berlin.

Strasser, Peter (2018): Spenglers Visionen. Hundert Jahre Untergang des Abendlandes. Wien.

Tanner, Jakob (2014): Die Schweiz im Grossen Krieg. Plädoyer für eine transnationale Geschichte. In: Rossfeld, Roman/Buomberger, Thomas/Kury, Patrick (Hg.): 14/18. Die Schweiz und der Grosse Krieg. Baden, S. 8-17.

Tanner, Jakob (2015): Geschichte der Schweiz im 20. Jahrhundert. München.

Tribelhorn, Marc (2018): Was bleibt von den Debatten um den Landesstreik? In: Neue Zürcher Zeitung, 24.11.2018; https://www.nzz.ch/feuilleton/was-bleibt-von-den-debatten-um-den-landesstreik-ld.1439167 [26.3.2019].

Tyack, David/Cuban, Larry (1995): Tinkering toward Utopia. A Century of Public School Reform. Cambridge, MA.

Zala, Sacha (2014): Krisen, Konfrontation, Konsens (1914-1949). In: Kreis, Georg (Hg.): Die Geschichte der Schweiz. Basel, S. 490-547.

Ziegler, Peter (1998): 200 Jahre Erziehungsrat des Kantons Zürich 1798 bis 1998. Zürich. 